\title{
BIOFEEDBACK: UM RECURSO TERAPÊUTICO PARA OS TRANSTORNOS DE ANSIEDADE ${ }^{1}$
}

\author{
BIOFEEDBACK: A THERAPEUTIC STRATEGY FOR ANXIETY DISORDERS
}

\author{
Denise Venturini Vidal' ${ }^{2}$ Andriza Corrêa ${ }^{3}$
}

\section{RESUMO}

A ansiedade, suas manifestações e sua complexidade retratam um dos maiores desafios enfrentados pela pesquisa e tratamento da saúde mental na contemporaneidade. Assim, estudos relacionados a esta temática são cada vez mais importantes. Inserindo-se na discussão, este estudo teve por objetivo compreender o biofeedback enquanto recurso terapêutico na perspectiva da terapia cognitivo-comportamental para os transtornos de ansiedade. A pesquisa foi realizada a partir de uma revisão integrativa da literatura, a qual compreendeu a leitura de artigos, teses, dissertações, livros e demais estudos publicados que disponibilizam texto completo em língua portuguesa, a partir da busca em bases de dados científicas em plataformas eletrônicas online. Foi realizada uma análise de conteúdo para a categorização dos estudos com o objetivo de organizar e sumarizar as informações de maneira concisa, formando um banco de dados de fácil acesso e manejo. No total, foram selecionados e analisados 10 estudos na íntegra, seguindo os critérios de inclusão e exclusão pré-estabelecidos. Neste processo, foi incluída a metodologia de busca por referências nas referências, para ampliar o número de estudos encontrados. Pesquisas apontam evidências positivas para a eficácia da utilização de equipamento de biofeedback como ferramenta terapêutica na redução da ansiedade do estresse e da sintomatologia associada. Ao final, entende-se que o biofeedback poderá oferecer aos profissionais da saúde uma nova técnica terapêutica, não invasiva, não medicamentosa, que torna a psicoterapia uma intervenção mais objetiva incorporada aos recursos terapêuticos já utilizados pela terapia cognitivo-comportamental.

Palavras-chave: Recurso terapêutico, processos fisiológicos, terapia cognitivo-comportamental.

\section{ABSTRACT}

Anxiety, its manifestation and complexity represent one of the biggest challenges faced by research and treatment of mental health in modern age. Therefore, studies related to this area are significant. This way, the objective of this study was to understand the biofeedback as a therapeutic tool in the perspective of cognitivebehavioral therapy to anxiety disorder. The research was conducted based on an integrative literature review, which involved reading articles, thesis, essays, books and studies that were published in congresses annals. These documents are offered in complete versions in Portuguese and were found through scientific database searches in online electronic platforms. A content analysis was done in order to categorize, organize and summarize the studies found, thus creating an accessible database. Altogether, a total of ten studies were fully read and analyzed following the predetermined inclusion and exclusion criteria. During this process, a method of searching in the references was included so that the number of studies found were increased. These studies show positive evidences related to the effectiveness of the biofeedback as therapeutic tool to reduce stress anxiety and associated symptomatology. At the end, it was concluded that biofeedback may offer to professionals of the area a new - non-invasive and non-drug - therapeutic technique that enables

${ }^{1}$ Revisão bibliográfica.

${ }^{2}$ Discente do curso de psicologia da Faculdade Integrada de Santa Maria - FISMA. E-mail: denise.venturini.vidal@gmail.com ${ }^{3}$ Orientadora, Doutora em psicologia, Especialista em Terapia Cognitivo-comportamental. Docente do curso de psicologia da Faculdade Integrada de Santa Maria - FISMA. E-mail: andrizascorrea@gmail.com 
psychotherapy to become a more straightforward intervention integrated to the therapeutic resources already in use by the cognitive-behavioral therapy.

Keywords: Therapeutic resource, physiological processes, cognitive-behavioral therapy.

\section{INTRODUÇÃO}

A complexidade dos transtornos de ansiedade continua atraindo a atenção de vários pesquisadores importantes na área da psicopatologia e da psicoterapia (CLARK; BECK, 2012; KNAPP; BECK, 2008; BECK, 1997; LEAHY, 2018). Nos últimos anos, ocorreu um crescimento nas pesquisas básica e aplicada sobre o processamento de informações sob a perspectiva do modelo cognitivo de ansiedade. A ansiedade é um elemento presente na sociedade contemporânea e a tenacidade de suas manifestações clínicas retrata um dos maiores desafios enfrentados pela pesquisa e pelo tratamento da saúde mental (CLARK; BECK, 2012).

A terapia cognitivo-comportamental é uma das abordagens terapêuticas no ramo da psicologia conhecida pela sua atuação prática e objetiva. Teve seu início na década de 1960, e seu precursor é Aaron Beck. Desde o seu surgimento, a TCC vem se destacando no tratamento de diversos transtornos mentais, entre eles, o transtorno de ansiedade e suas especificações (ALMEIDA et al., 2016).

Na perspectiva da TCC, um dos recursos utilizados que tem ganho destaque no tratamento dos transtornos de ansiedade é o biofeedback. Este recurso pode ser considerado um método que proporciona retorno imediato de processos fisiológicos, em que o paciente pode não estar consciente ou apresentar dificuldades para controlar. Além disso, permite o aprendizado da regulação voluntária de respostas fisiológicas e emocionais e é utilizado como método de autorregulação dos processos corporais, musculares, respiratórios e cognitivos (DONNER, 2011).

De acordo com o Manual Diagnóstico e Estatístico de Transtornos Mentais - DSM-5, a ansiedade é uma antecipação de ameaça à resposta emocional ao perigo futuro e comportamentos de cautela ou esquiva. Frequentemente está associada à tensão muscular, à vigilância e à preparação para luta ou fuga (APA, 2014).

Os transtornos de ansiedade se diferenciam entre si e são divididos em: transtornos de ansiedade de separação, mutismo seletivo, fobia específica, transtorno de ansiedade social ou fobia social, transtorno do pânico, agorafobia, transtorno de ansiedade generalizada, transtorno de ansiedade induzido por substância/medicamento, transtorno de ansiedade devido à outra condição médica, transtorno de ansiedade especificado e não especificado. Cada uma dessas patologias supracitadas apresenta critérios diagnósticos específicos que incluem e compartilham características de medo e ansiedade excessiva e perturbações comportamentais relacionadas. Os tipos de objetos ou situações 
que induzem comportamento de esquiva, por conteúdo dos pensamentos ou por crenças com frequência induzidas pelo estresse diferem entre si (APA, 2014).

Como o recurso do biofeedback é um campo genuinamente multidisciplinar com o objetivo de auxiliar os pacientes a desenvolver consciência, confiança e capacidade para o autocontrole (DONNER, 2011), merece assim ser explorado e esclarecido aos profissionais que atuam na área clínica e demais interessados. Acrescenta-se a esta perspectiva os transtornos de ansiedade, que merecem atenção clínica e um diagnóstico preciso pelo seu quadro persistente e pela capacidade de interferir em diferentes áreas importantes da vida do indivíduo, como a social, profissional e pessoal.

Assim, este estudo se propõe a elucidar o biofeedback como recurso para auxiliar no tratamento dos transtornos de ansiedade, considerando as principais características das preocupações excessivas que afetam de forma significativa o funcionamento psicossocial do indivíduo (APA, 2014), bem como o trabalho terapêutico realizado com base no modelo cognitivo, abordando estratégias e técnicas com ênfase no biofeedback. Pretendeu-se levantar informações que poderão orientar intervenções para o trabalho clínico do psicólogo.

Tendo em vista esses aspectos, propõe-se compreender o biofeedback enquanto recurso terapêutico na perspectiva da TCC para os transtornos de ansiedade. Além disso, a verificar as contribuições que o biofeedback pode trazer para o paciente com esse diagnóstico, e compreender a técnica do biofeedback utilizada por profissionais da psicologia como recurso terapêutico que torna possível o controle voluntário e a autorregulação dos sintomas de ansiedade.

\section{MATERIAL E MÉTODOS}

\section{TIPO DE ESTUDO}

Para a elaboração deste estudo, foi realizada uma revisão integrativa da literatura que é considerada uma ampla abordagem metodológica referente a revisões. Neste sentido, gera um panorama consistente de maneira compreensível de conceitos complexos, teorias ou problemas de saúde relevantes. Esse tipo de revisão permite a construção de uma análise, contribuindo para discussões sobre métodos e resultados de pesquisas, assim como reflexões sobre a realização de novas pesquisas (SOUZA; SILVA; CARVALHO, 2010).

A seguir, são apontadas as seis fases do processo de elaboração da revisão integrativa: 1) elaboração da pergunta norteadora; 2) busca na literatura; 3) coleta de dados; 4) análise crítica dos estudos incluídos; 5) discussão dos resultados; 6) apresentação da revisão integrativa (SOUZA; SILVA; CARVALHO, 2010). 


\section{PROCEDIMENTO}

Foram coletados artigos, teses, dissertações e livros e demais estudos científicos publicados que disponibilizam texto completo em língua portuguesa, a partir de busca em bases de dados científicas em plataformas eletrônicas online, como SciELO, LILACS, PePSIC e BVS-Psi. Optou-se pela não delimitação de ano de publicação, pois, por via deste instrumento de pesquisa, verificou-se que os estudos deste tema são pouco encontrados na literatura brasileira. Serviram como descritores para coleta de dados os seguintes: biofeedback; transtornos de ansiedade; terapia cognitivo-comportamental.

Nesta busca, os critérios de inclusão definidos previamente foram: estudos que abordam o biofeedback como um recurso terapêutico e estudos que abordam o biofeedback para os transtornos de ansiedade utilizado em terapia cognitivo-comportamental. Os critérios de exclusão para a seleção dos estudos foram: estudos em outro idioma e estudos que tivessem outras abordagens terapêuticas. Assim, a busca restringiu-se aos trabalhos publicados somente em português, que estavam disponíveis na íntegra gratuitamente. Também foram eliminados estudos que não retratam a temática referente ao biofeedback como um recurso terapêutico.

Em um primeiro momento, após a leitura dos títulos dos 32 estudos encontrados, foram eliminados 15. Em um segundo momento, foi realizada a leitura de 17 resumos. Por fim, foram lidos e analisados 10 estudos na íntegra, seguindo os critérios de inclusão e exclusão pré-estabelecidos. Neste processo, foi incluída a metodologia de busca por referências nas referências (snowball sampling) para ampliar o número de estudos encontrados.

\section{ANÁLISES DOS DADOS}

Após formar um banco de dados de fácil acesso e manejo com os estudos selecionados, com o objetivo de organizar e sumarizar as informações de maneira concisa, foi realizada análise de conteúdo para a categorização de tais estudos. A análise de conteúdo caracteriza-se como um método que, histórica e cotidianamente, produz sentidos e significados na diversidade de amostragem presentes no mundo acadêmico (BARDIN, 2016).

Os achados foram apreciados individualmente constituindo as etapas finais da revisão integrativa. Por fim, foi realizado um delineamento dos eixos e tendências mais salientes no conjunto do material encontrado, procurando explicações para os resultados diferentes ou conflitantes nos diferentes estudos. Os estudos selecionados tiveram uma análise detalhada para, desta forma, garantir a validade da revisão integrativa. 


\section{RESULTADOS E DISCUSSÃO}

Esta seção apresenta dados da pesquisa obtidos por meio de revisão da literatura. Eles foram encontrados nas buscas em bases de dados e também através da busca por referências nas referências. Desse modo, serão apresentados na Tabela 1 os resultados desta revisão com o título da publicação, autor, local e ano, objetivo e principais resultados. Salienta-se que os textos utilizados nesta tabela foram utilizados tal como constam nos estudos verificados.

Tabela 1 - Sumarização dos achados.

\begin{tabular}{lccl}
\hline \multicolumn{1}{c}{ Título } & Autores & Local/Ano & \multicolumn{1}{c}{ Objetivo } \\
\hline $\begin{array}{l}\text { Eficácia do biofeedback } \\
\text { na redução da ansiedade - } \\
\text { sua potencialidade como } \\
\begin{array}{l}\text { intervenção do especialista } \\
\text { em enfermagem de saúde }\end{array}\end{array}$ & Moraes, H; & Portugal & $\begin{array}{l}\text { Verificar evidências científicas que } \\
\text { Vustentam a eficácia dos progra- }\end{array}$ \\
mental e psiquiátrico & Lopes, R. & 2018 & $\begin{array}{l}\text { mas de biofeedback na redução da } \\
\text { ansiedade }\end{array}$ \\
$\begin{array}{l}\text { Emprego do biofeedback } \\
\text { no tratamento de doenças } \\
\text { crônicas }\end{array}$ & Domingos, N. A. & Brasil & Analisar criticamente a literatura \\
& M.; Miyazaki, & 2017 & $\begin{array}{l}\text { científica, sobre o uso e a eficácia } \\
\text { do biofeedback no tratamento de }\end{array}$ \\
& M. C. O. S. & & doenças crônicas.
\end{tabular}

$\begin{array}{lccl}\text { Biofeedback em terapia } & \text { Neves Neto, } & \text { Brasil, } & \begin{array}{l}\text { Descrever a utilização do biofee- } \\ \text { cognitivo-comportamental }\end{array} \\ \text { A.R. } & 2010 & \begin{array}{l}\text { dback como um recurso terapêuti- } \\ \text { co complementar à psicoterapia. }\end{array}\end{array}$

Muitas doenças foram foco de atenção nos estudos selecionados, mas, apenas dois estudos nacionais. Estudos estes com uma variabilidade de idade dos participantes. Isso indica que o biofeedback é uma ferramenta que pode ser utilizada numa ampla gama de indivíduos. O Biofeedback poderá oferecer aos profissionais da saúde mental uma nova ferramenta terapêutica, não invasiva, não medicamentosa, que tem o potencial de ampliar o conhecimento sobre corpo e mente, tornando a psicoterapia uma intervenção mais objetiva.

Biofeedback e ansiedade no ensino superior: comparação de eficácia entre dos programas breves

\begin{tabular}{|c|c|c|}
\hline $\begin{array}{l}\text { Biofeedback no tratamento } \\
\text { de transtorno relacionados } \\
\text { ao estresse e a ansiedade: }\end{array}$ & $\begin{array}{l}\text { Lantyer, A, S. } \\
\text { Viana, M. B.; } \\
\text { Padovani, R. C. }\end{array}$ & $\begin{array}{c}\text { Brasil, } \\
2013\end{array}$ \\
\hline $\begin{array}{l}\text { O estresse e a ansiedade } \\
\text { aos exames: Contributo do }\end{array}$ & $\begin{array}{c}\text { Rodrigues,H. ; } \\
\text { Pereira, A. }\end{array}$ & $\begin{array}{c}\text { Portugal, } \\
2010\end{array}$ \\
\hline
\end{tabular}

biofeedback

Mateus, M.

\section{Chaló, P. Pereira, \\ Portugal, \\ Comparar a eficácia entre dois \\ A. Sancho, L. 2016 programas breves de biofeedback.}

Analisar criticamente a literatura científica sobre o uso e a eficácia do biofeedback como tratamento ou intervenção terapêutica nos transtornos relacionados ao estresse e à ansiedade.

Estudar o biofeedback enquanto ferramenta auxiliar e complementar com intervenções cognitivas e comportamentais, as sensoriais e as intervenções interpessoais.
Os resultados mostram que a intervenção com o biofeedback em contexto universitário permite uma redução da ansiedade dos alunos. Os resultados demostram que técnicas de biofeedback são eficazes no manejo do estresse e da ansiedade nas diferentes populações estudadas.

Estudos apontam que, o biofeedback evidencia eficácia enquanto técnica complementar no controle do estresse em contexto universitário. 


\begin{tabular}{|c|c|c|c|c|}
\hline $\begin{array}{l}\text { Técnicas de respiração para } \\
\text { a redução do estresse em } \\
\text { terapia cognitivo-compor- } \\
\text { tamental }\end{array}$ & $\begin{array}{c}\text { Neves Neto, } \\
\text { A. R. }\end{array}$ & $\begin{array}{l}\text { Brasil, } \\
2011\end{array}$ & $\begin{array}{l}\text { Revisar as técnicas de respiração } \\
\text { aplicada a redução do estresse e } \\
\text { aumento do bem estrar biopsicos- } \\
\text { social. }\end{array}$ & $\begin{array}{l}\text { As técnicas de respiração devem } \\
\text { ser foco de estudos e de aplicação } \\
\text { práticas na saúde, de profissionais } \\
\text { competentes e com treinamento } \\
\text { nas bases anatomofisiológicas e } \\
\text { psicológicas da respiração, capazes } \\
\text { de instruírem seus pacientes de } \\
\text { forma segura e eficaz. }\end{array}$ \\
\hline $\begin{array}{l}\text { Estudo de ansiedade em } \\
\text { alunos do ensino superior } \\
\text { utilizando o biofeedback }\end{array}$ & Ribeiro,L.N.S. & $\begin{array}{c}\text { Portugal, } \\
2012\end{array}$ & $\begin{array}{l}\text { Caracterizar os estudantes quanto } \\
\text { aos seus níveis de ansiedade, fatores } \\
\text { de estresse e bem-estar e a relação } \\
\text { destes com variáveis sociodemo- } \\
\text { gráficas e académicas (estudo tipo } \\
\text { transversal); 2) avaliar a eficácia da } \\
\text { intervenção na gestão da ansiedade, } \\
\text { com programa de Biofeedback e } \\
\text { comparar com a Terapia Cognitivo } \\
\text { Comportamental - TCC; 3) Estudar } \\
\text { e avaliar a intervenção específica } \\
\text { com biofeedback }\end{array}$ & $\begin{array}{l}\text { Os resultados mostraram que o } \\
\text { biofeedback é particularmente } \\
\text { eficaz na redução dos níveis ele- } \\
\text { vados de ansiedade e estresse nos } \\
\text { estudantes. }\end{array}$ \\
\hline $\begin{array}{l}\text { Impacto do biofeedback } \\
\text { nos níveis de estresse, } \\
\text { ansiedade e autorregulação } \\
\text { emocional em estudantes } \\
\text { universitários. }\end{array}$ & Costa, V.A.R & $\begin{array}{c}\text { Portugal } \\
2016\end{array}$ & $\begin{array}{l}\text { Este estudo pretende avaliar a } \\
\text { eficácia do biofeedback na redução } \\
\text { dos níveis de ansiedade e estresse } \\
\text { e na melhoria da autorregulação } \\
\text { emocional. Enquanto técnica de } \\
\text { terapêutica não invasiva. }\end{array}$ & $\begin{array}{l}\text { Os resultados alcançados no pré e } \\
\text { pós-teste através do qual se verificou } \\
\text { uma redução estatisticamente sig- } \\
\text { nificativa do estresse em ambos os } \\
\text { grupos e uma redução da ansiedade } \\
\text { somente no grupo sujeito ao biofee- } \\
\text { dback. Relativamente à autorregula- } \\
\text { ção emocional apesar de se ter veri- } \\
\text { ficado melhorias com o biofeedback } \\
\text { estas não foram significativas }\end{array}$ \\
\hline $\begin{array}{l}\text { Biofeedback na prevenção } \\
\text { da ansiedade em alunos } \\
\text { universitários. }\end{array}$ & Chaló, P. A. F. & $\begin{array}{c}\text { Portugal } \\
2013\end{array}$ & $\begin{array}{l}\text { Realizar um estudo exploratório } \\
\text { para verificar a eficácia do biofee- } \\
\text { dback na redução e prevenção da } \\
\text { ansiedade e estresse em alunos } \\
\text { que frequentam o primeiro ano da } \\
\text { universidade e também verificar a } \\
\text { potencialidade deste instrumento } \\
\text { na utilização como treino preven- } \\
\text { tivo no desenvolvimento destas } \\
\text { problemáticas. }\end{array}$ & $\begin{array}{l}\text { O resultados revelam bons indi- } \\
\text { cadores das potencialidades da } \\
\text { intervenção com biofeedback na } \\
\text { redução da ansiedade e redução } \\
\text { de estresse. }\end{array}$ \\
\hline
\end{tabular}

Foram encontrados um total de 10 estudos através da busca nas bases de dados. A maioria destes estudos (60\% da amostra) foi produzida em Portugal entre os anos de 2010 e 2018, o que totalizou seis estudos incluídos. No Brasil, foram produzidos quatro estudos ( $40 \%$ da amostra) relacionados ao tema, publicados entre 2010 e 2012.

No que se refere aos participantes de cada programa de intervenção com o biofeedback, foram encontrados em sua maioria estudos realizados com estudantes universitários (CHALÓ, et al. 2016; COSTA, 2016; CHALÓ, 2013; RODRIGUES; PEREIRA, 2010; RIBEIRO, 2012) que corresponde a 
$50 \%$ dos achados. Segundo estes estudos, os estudantes são considerados indivíduos com elevados níveis de ansiedade e estresse, o que justifica o interesse dos pesquisadores nesta população.

Também foi encontrado um estudo que aborda o biofeedback como intervenção com especialista em enfermagem de saúde mental e psiquiátrica (MORAIS; VICENTE; LOPES, 2018). Um sobre o emprego do biofeedback no tratamento de doenças crônica (DOMINGOS; MIYAZAKI, 2017). Dois estudos que abordam o biofeedback em terapia cognitivo-comportamental (NEVES NETO, 2018a; NEVES NETO, 2018b). Já sobre o biofeedback e a ansiedade, foram selecionados cinco estudos que se debruçam sobre as temáticas analisadas no âmbito do ensino superior (CHALÓ et al., 2016; COSTA, 2016; CHALÓ, 2013; PEREIRA, 2010; RODRIGUES; PEREIRA, 2010; RIBEIRO, 2012). Finalmente, um estudo sobre biofeedback no tratamento de estresse e ansiedade (LANTYER; VIANA; PADOVANI, 2013).

\section{BIOFEEDBACK NA TERAPIA COGNITIVO-COMPORTAMENTAL}

O estudo de Neves Neto (2018a) teve como objetivo descrever a utilização do biofeedback como um recurso terapêutico complementar à psicoterapia. Os resultados mostram que a ferramenta do biofeedback, associada às técnicas da terapia cognitivo-comportamental são eficazes, e salienta a relação entre estados cognitivos, afetivos, comportamentais e alterações do sistema nervoso autônomo nas modificações das reações autonômicas através da aprendizagem de forma consciente.

No trabalho de Rodrigues e Pereira (2010), realizado em Portugal sobre a contribuição do biofeedback para o estresse e a ansiedade frente aos exames acadêmicos, objetivou-se estudar este recurso enquanto ferramenta auxiliar e complementar com intervenções cognitivas e comportamentais. Os resultados do estudo apontam que o biofeedback evidencia sua eficácia enquanto técnica complementar no controle do estresse em contexto universitário. Além disso, os autores sugerem que o biofeedback apresenta inúmeros aspectos positivos comparativamente a outras formas de tratamento. Este recurso não apresenta efeitos colaterais como os medicamentos, não é invasivo, é seguro e indolor. O paciente participa do processo de forma consciente e ativo no transcurso de modificações de hábitos e comportamentos (RODRIGUES; PEREIRA, 2010; NEVES NETO, 2018b).

Pode-se dizer que o biofeedback é visto como um processo psicoeducacional em terapia cognitivo-comportamental, que utiliza sofisticados equipamentos eletrônicos capazes de monitorar os sinais vitais relevantes, e ainda revisa as técnicas de respiração aplicada a redução do estresse e aumento do bem-estar biopsicossocial. Com ele, é possível revisar as técnicas de respiração aplicada à redução do estresse e aumento do bem-estar biopsicossocial (NEVES NETO, 2018b).

Considera-se que os resultados dos estudos sobre as técnicas de respiração devem ser foco de estudos e de aplicações práticas na saúde, por profissionais competentes e com treinamento nas bases anatomofisiológicas e psicológicas da respiração, capazes de instruírem seus pacientes de forma 
segura e eficaz. Neves Neto (2018b) destaca que a medicina e a psicologia contemporânea têm uma importante contribuição ao investigar mecanismos psicofisiológicos de práticas voltadas à redução do estresse e de outras patologias. Tanto a terapia cognitivo-comportamental e a medicina comportamental, quanto o biofeedback podem ser associados às técnicas de respiração, visando a uma sinergia entre diferentes procedimentos terapêuticos (NEVES NETO, 2011).

Desde os primeiros estudos do psicólogo Neal Miller abriu-se um novo campo de pesquisas das relações corpo-mente denominado por psicofisiologia. Desta forma, o biofeedback pode oferecer aos profissionais da saúde uma nova técnica terapêutica, não invasiva, não medicamentosa. Assim podendo tornar a psicoterapia uma intervenção mais objetiva incorporada aos recursos terapêuticos já utilizados pela terapia cognitivo-comportamental (NEVES NETO, 2018a).

\section{BIOFEEDBACK PARA TRANSTORNOS DE ANSIEDADE}

O estudo de Morais et al. (2018), que analisa a eficácia do biofeedback na redução da ansiedade, apresenta uma revisão integrativa da literatura publicada em Portugal. Este estudo refere-se à intervenção do especialista em enfermagem de saúde mental e psiquiátrica. Foram analisados trabalhos científicos publicados entre 2011 e 2016 com o objetivo de verificar se existem evidências científicas que sustentam a eficácia dos programas de biofeedback na redução da ansiedade. Os autores concluem que a maioria das pesquisas consultadas demonstrou a eficácia da utilização do biofeedback como ferramenta terapêutica na redução da ansiedade e da sintomatologia associada. Também consideram que o procedimento é eficaz na redução dos sintomas de ansiedade nas diferentes populações estudadas.

Através de uma revisão crítica da literatura científica, Lantyer, Viana e Padovani (2013) investigaram sobre o uso e a eficácia do biofeedback como tratamento ou intervenção terapêutica nos transtornos relacionados ao estresse e a ansiedade. Nesta revisão, foram incluídas pesquisas com ensaios clínicos randomizados e revisões, entre os anos de 2008 a 2012 com o tema biofeedback, estresse e ansiedade. Os resultados corroboram com os demais autores já citados, que técnicas de biofeedback são eficazes no manejo do estresse e da ansiedade nas diferentes populações estudadas (pacientes psiquiátricos, estudantes universitários e de ensino médio, pacientes cardíacos, militares, entre outros.). A grande maioria dos estudos consultados pelos autores demostra a eficácia da utilização de equipamento de biofeedback como recurso terapêutico no manejo da ansiedade e do estresse e de transtornos relacionados.

No estudo de Chaló et al. (2016), foi realizada uma comparação da eficácia entre os programas breves e a ansiedade no ensino superior através do biofeedback. Os autores concluíram que a intervenção com o biofeedback em contexto universitário permite uma redução da ansiedade dos alunos, estando em convergência com resultados semelhantes em outros estudos internacionais. 
Em uma revisão sistemática da literatura de estudos publicados dentre os anos de 2009 a 2014, Domingos e Miyazaki (2017) propuseram verificar a utilização do biofeedback no tratamento de doenças crônicas em participantes com uma variabilidade de idade. Os resultados apresentados demostram que há indicações de evidências positivas no uso do biofeedback em muitas doenças que foram foco de atenção nos estudos selecionados. Isso indica que o biofeedback é uma ferramenta que pode ser utilizada numa ampla gama de indivíduos em diferentes faixas etárias.

No que se refere aos cinco estudos realizados com estudantes universitários, todos realizados em Portugal, quatro deles utilizaram o State Trait Anxiety Inventory (STAI), o Inventário de Estresse nos Estudantes Universitários (ISEU) o Teste de Orientação de Vida (LOT, Life Orientation Test), o Patient's Health Questionnaire (PHQ-9) e os Termómetros Emocionais (TE). Como instrumento de avaliação das variáveis psicofisiológicas foi usado o Biofeedback 2000x-pert (CHALÓ, et al. 2016; COSTA 2016; CHALÓ 2013; RODRIGUES; PEREIRA, 2010; RIBEIRO, 2012).

O Biofeedback 2000x-pert é um equipamento portátil cujos módulos permitem recolher sinais da superfície da pele, através de sensores não invasivos. Este instrumento reconhece os seguintes sinais: atividade eletrodérmica, temperatura corporal, aumento da frequência motora, alterações na circulação sanguínea e atividade muscular. Com estas informações, permite-se ao indivíduo uma maior tomada de consciência sobre esses processos e a aquisição de um maior controle sobre o corpo e a mente (CHALÓ, 2013; COSTA, 2016).

Neste contexto de estudantes universitários, os resultados no pré e pós-teste apontam para uma diminuição significativa nos níveis de ansiedade e estresse. Além disso, revelam bons indicadores das potencialidades da intervenção com biofeedback. Em contraponto, os autores sugerem que é possível identificar que são necessários estudos futuros para dar continuidade ao trabalho que tem se desenvolvido a nível de intervenção com o biofeedback (CHALÓ, 2013; CHALÓ et al., 2016; COSTA, 2016; RIBEIRO, 2012).

\section{CONSIDERAÇÕES FINAIS}

Este estudo teve como objetivo compreender o biofeedback enquanto recurso terapêutico na perspectiva da terapia cognitivo-comportamental para os transtornos de ansiedade. Além disso, verificar as contribuições que o biofeedback pode trazer para o paciente com diagnóstico de transtorno de ansiedade, e compreender a técnica do biofeedback utilizada por profissionais da psicologia como uma ferramenta, que torna possível o controle voluntário dos sintomas e a autorregulação.

É possível perceber que o biofeedback é um recurso terapêutico inovador que possui aspectos fundamentais para prática e proporciona benefícios a serem concedidos para o paciente de forma a 
melhorar sua qualidade de vida. Além disso, é de grande relevância que o terapeuta tenha conhecimento e identifique características de cada paciente para poder entender as variáveis do meio em que ele está inserido que podem interferir no tratamento.

Desta forma, destaca-se a importância de clínicos e profissionais da saúde atentarem para a incidência de quadros de ansiedade, geralmente em comorbidades com outros transtornos. Isso produz reflexões diante da seriedade do trabalho a ser desenvolvido pelo psicólogo, tanto na saúde mental, quanto na saúde pública e privada, em clínicas e instituições entre outros campos de atuação.

Destaca-se também a relevância do olhar humanizado, visando melhorar a qualidade de vida das pessoas em sofrimento psíquico. Cabe salientar também que a literatura estrangeira sobre o assunto é vasta, entretanto, publicações no Brasil são escassas. Todos os estudos consultados apontam evidências positivas para a eficácia da utilização de equipamento de biofeedback como ferramenta terapêutica na redução da ansiedade do estresse e da sintomatologia associada. Contudo, sugere-se mais estudos sobre o uso do biofeedback como ferramenta útil na intervenção com pacientes com diagnóstico de transtornos de ansiedade.

Por fim, espera-se que este estudo possa ser usado como um aporte para a construção de novos trabalhos. Além disso, como mais um contributo à incessante procura partilhada por psicólogos e outros profissionais de saúde de conhecer o ser humano em toda sua complexidade, explorando formas alternativas não invasivas, como o instrumento do biofeedback, para a redução da sintomatologia dos transtornos de ansiedade e de melhorias da autorregulação emocional dos processos fisiológicos.

\section{REFERÊNCIAS}

ALMEIDA, R. S. et al. O uso dos grupos terapêuticos no tratamento dos transtornos de ansiedade sob o olhar da terapia cognitivo-comportamental. Ciências Biológicas e da Saúde, v. 3, p. 101-118. Maceió, 2016. Disponível em: https://bit.ly/2ITFodg. Acesso em: 07 abr. 2019.

\section{AMERICAN PSYCHOLOGY ASSOCIATION. Manual diagnóstico e estatístico de transtornos mentais - DSM-5. 5. ed. Porto Alegre: Artmed, 2014.}

BARDIN, L. Análise de conteúdo. 3. ed. São Paulo: Edições 70, 2016.

BECK, J. S. Terapia cognitiva: teoria e prática. Porto Alegre: Artmed, 1997.

CLARK, D. A.; BECK, A. T. Terapia cognitiva para os transtornos de ansiedade. Porto Alegre: Artmed, 2012. 
COSTA, V. A. R. Impacto do biofeedback nos níveis de estresse, ansiedade e autorregulação emocional em estudantes universitários. 2016. [Dissertação Mestrado em Psicologia] - Departamento de Educação e Psicologia, Universidade de Aveiro, Aveiro: 2016.

CHALÓ, P. A. F. Biofeedback na prevenção da ansiedade em alunos universitários. 2013. [Dissertação Mestrado em Psicologia] - Departamento de Educação e Psicologia, Universidade de Aveiro, Aveiro: 2013.

CHALÓ, P. et al. Biofeedback e ansiedade no ensino superior: comparação da eficácia entre dois programas breves. Psicologia, Saúde \& Doenças, v. 17, n. 1, p. 60-66, 2016. Disponível em: https:// bit.ly/2ISDqtA. Acesso em: 28 set. 2019.

DOMINGOS, N. A. M; MIYAZAKI, M. C. O. S. Emprego do biofeedback no tratamento de doenças crônicas. Arquivos de Ciências da Saúde, v. 24, n. 1, p. 15-22, 2017. Disponível em: https://bit.ly/37nswW5. Acesso em: 27 set. 2019.

DONNER, O. I. Biofeedback. In: RANGÉ, B. et al. Psicoterapias cognitivo - comportamentais: um diálogo com a psiquiatria. 2. ed. Porto Alegre: Artmed, 2011. p 222-237.

KNAPP, P. ; BECK, A. T. Fundamentos, modelos conceituais, aplicações e pesquisa da terapia cognitiva. Revista Brasileira de Psiquiatria. v. 30, p. s54-s64, 2008.

LANTYER, A. S; VIANA, M. B; PADOVANI, R. C. Biofeedback no tratamento de transtornos relacionados ao estresse e à ansiedade: uma revisão crítica. Psico-USF, Itatiba, p. 131-140. 2013. Disponível em: https://bit.ly/3mq5mmc. Acesso em: 27 set. 2019.

LEAHY, R. L. Técnicas de terapia cognitiva: Manual do terapeuta. Porto Alegre: Artmed, 2018.

MORAIS, H. et al. Eficácia do biofeedback na redução da ansiedade - sua potencialidade como intervenção do especialista em enfermagem de saúde mental e psiquiátrica. Revista Investigação em Enfermagem. n. 22. Série 2-fev. 2018, p. 9. Disponível em: https://bit.ly/3r4ewIU. Acesso em: 28 set. 2019.

NEVES NETO, A. R. Biofeedback em terapia cognitivo-comportamental. Arquivos Médicos dos Hospitais e da Faculdade de Ciências Médicas da Santa Casa de São Paulo, v. 55, n. 3, p. 127-132, 2018a. 
NEVES NETO, A. R. Técnicas de Respiração para a redução do estresse em terapia cognitivo-comportamental. Arquivos Médicos dos Hospitais e da Faculdade de Ciências Médicas da Santa Casa de São Paulo, v. 56, n. 3, p. 158-168, 2018 b.

PEREIRA, M.; RANGÉ, B. P. Terapia cognitiva. In: RANGÉ, B. et al. Psicoterapias cognitivo-comportamentais: um diálogo com a psiquiatria. 2. ed. Porto Alegre: Artmed, 2011. p. 20-32.

RIBEIRO, L. N. S. Estudo da ansiedade em alunos do ensino superior utilizando o biofeedback. 2012. [Tese Doutorado em Psicologia] - Departamento de Educação e Psicologia, Universidade de Aveiro, Aveiro: 2013. p. 328.

RODRIGUES, H.; PEREIRA, A. O stress e a ansiedade aos exames: contributo de biofeedback. In: PEREIRA, A.S.; CASTANHEIRA, H.; MELO, A.B.; FERREIRA, A. I.; VAGOS, P. Apoio psicológico no ensino superior: modelos e práticas: Actas do I Congresso Nacional da RESAPES-AP. Universidade de Aveiro. Aveiro: 2010. p. 89-95.

SOUZA, M. T.; SILVA, M. D.; CARVALHO, R. Revisão integrativa: o que é e como fazer. Einstein (São Paulo), São Paulo, v. 8, n.1, p. 102-106, Mar. 2010. Disponível em: https://bit.ly/2LHyskB. Acesso em: 07 maio 2019. 